\title{
Efficacy and resistance of different artemisinin-based combination therapies: a systematic review and network meta-analysis
}

\author{
Peterson Gitonga Mathenge ${ }^{\mathrm{a}, \mathrm{b}, 1}$, Soon Khai Low ${ }^{\mathrm{c}, \mathrm{d}, 1}$, Nguyen Lam Vuong ${ }^{\mathrm{c}, \mathrm{e}}$, \\ Muawia Yousif Fadlelmola Mohamed ${ }^{\mathrm{c}, \mathrm{f}}$, Hazem Abdelkarem Faraj ${ }^{\mathrm{c}, g}$, Ghada Ibrahim Alieldin ${ }^{\mathrm{c}, \mathrm{h}}$, \\ Rawan Al khudari ${ }^{c, i}$, Nusaiba Adam Yahia ${ }^{c, j}$, Adnan Khan ${ }^{\mathrm{c}, \mathrm{k}}$, Omar Mohammad Diab ${ }^{\mathrm{c}, \mathrm{l}}$, \\ Yara Mahmoud Mohamed ${ }^{\mathrm{c}, \mathrm{m}}$, Ahmad Helmy Zayan ${ }^{\mathrm{c}, \mathrm{n}}$, Gehad Mohamed Tawfik ${ }^{\mathrm{c}, \mathrm{o}}$, \\ Nguyen Tien Huy ${ }^{\mathrm{p}, \mathrm{q}, \mathrm{r}, * *}$, Kenji Hirayama ${ }^{\mathrm{s}, *}$ \\ ${ }^{a}$ Leading program, Graduate School of Biomedical Sciences, Nagasaki University, Nagasaki, Japan \\ ${ }^{\mathrm{b}}$ Department of Immunogenetics, Institute of Tropical Medicine (NEKKEN), Nagasaki University, Nagasaki, Japan \\ ${ }^{\mathrm{c}}$ Online Research Club, Japan \\ ${ }^{\mathrm{d}}$ School of Medicine, Faculty of Health and Medical Sciences, Taylor's University, Selangor, Malaysia \\ ${ }^{\mathrm{e}}$ University of Medicine and Pharmacy at Ho Chi Minh City, Ho Chi Minh City, Vietnam \\ ${ }^{\mathrm{f}}$ Faculty of Medicine, University of Khartoum, Sudan \\ ${ }^{\mathrm{g}}$ Faculty of Medicine, University of Tripoli, Tripoli, Libya

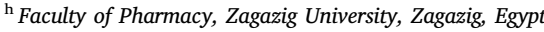 \\ ${ }^{\mathrm{i}}$ Faculty of Medicine, Damascus University, Damascus, Syria \\ ${ }^{\mathrm{j}}$ Faculty of Medicine, Omdurman Islamic University, Khartoum, Sudan \\ ${ }^{\mathrm{k}}$ Paediatrics, Rehman Medical Institute, Peshawar, Pakistan \\ ${ }^{1}$ Jordan University of Science and Technology, Irbid, Jordan \\ ${ }^{\mathrm{m}}$ Faculty of Medicine, Misr University for Science and Technology, Cairo, Egypt \\ ${ }^{n}$ Department of Otolaryngology, Menoufia University, Menoufia, Egypt \\ ${ }^{\circ}$ Faculty of Medicine, Ain Shams University, Cairo, Egypt \\ ${ }^{\mathrm{p}}$ Evidence Based Medicine Research Group, Ton Duc Thang University, Ho Chi Minh City 70000, Viet Nam \\ ${ }^{\mathrm{q}}$ Faculty of Applied Sciences, Ton Duc Thang University, Ho Chi Minh City 70000, Viet Nam \\ ${ }^{\mathrm{r}}$ Department of Clinical Product Development, Institute of Tropical Medicine (NEKKEN), School of Tropical Medicine and Global Health, Nagasaki University, Nagasaki \\ 852-8523, Japan \\ ${ }^{\mathrm{s}}$ Department of Immunogenetics, Institute of Tropical Medicine (NEKKEN), Leading Graduate School Program, Graduate School of Biomedical Sciences, Nagasaki \\ University, Sakamoto, Nagasaki, Japan
}

\section{A R T I C L E I N F O}

\section{Keywords:}

Malaria

Resistance

Artemisinin combination therapy

Efficacy

Recrudescence

Treatment failure

\begin{abstract}
A B S T R A C T
Background: Malaria parasites have developed resistance to most of the known antimalarial drugs in clinical practice, with reports of artemisinin resistance emerging in South East Asia (SEA). We sort to find the status of artemisinin resistance and efficacy of different modalities of the current artemisinin-based combination therapies (ACTs).

Methods: We carried out a systematic search in 11 electronic databases to identify in vivo studies published between 2001 and 2017 that reported artemisinin resistance. This was then followed by A network meta-analysis to compare the efficacy of different ACTs. Quality assessment was performed using the Cochrane Risk of Bias (ROB) tool for randomized controlled trials and National Institute of Health $(\mathrm{NIH})$ tool for cross-sectional studies. The study protocol was registered in PROSPERO under number CRD42018087574.

Results: With 8400 studies initially identified, 82 were eligible for qualitative and quantitative analysis. Artemisinin resistance was only reported in South East Asia. K13 mutation C580Y was the most abundant mutation associated with resistance having an abundance of $63.1 \%$ among all $\mathrm{K} 13$ mutations reported. Although the overall network meta-analysis had shown good performance of dihydroartemisinin piperaquine in the early years, a subgroup analysis of the recent years revealed a poor performance of the drug in relation to
\end{abstract}

\footnotetext{
* Corresponding author.

** Corresponding author at: Evidence Based Medicine Research Group, Faculty of Applied Sciences, Ton Duc Thang University, Ho Chi Minh City, Viet Nam.

E-mail addresses: nguyenlamvuong@ump.edu.vn (N.L. Vuong), nguyentienhuy4@duytan.edu.vn (N.T. Huy), hiraken@nagasaki-u.ac.jp (K. Hirayama).

${ }^{1}$ Authors equally contributed to the work.
} 
recrudescence, clinical failure and parasitological failure especially in the artemisinin resistant regions. Conclusion: With report of high resistance and treatment failure against the leading artemisinin combination therapy in South East Asia, it is imperative that a new drug or a formulation is developed before further spread of resistance.

\section{Introduction}

Malaria is a mosquito-borne disease caused by Plasmodium parasites that can be potentially fatal. World Health Organization (WHO) approximated that there were 216 million cases of malaria and 445,000 deaths from malaria worldwide in 2016 , with the highest global burden being reported in African region[1]. History of antimalarial resistance dates back in time, with resistance to quinine and chloroquine being recorded in the early and mid-1960s [2-5] in South East Asia (SEA) and South America. This necessitated the need of a new antimalarial drug and it was not until 1972 when artemisinin was discovered by Youyou Tu [6]. Artemisinin is a fast acting drug that targets blood-stage Plasmodium falciparum [7] but its efficacy when used as monotherapy is limited by its short half-life [8]. Artemisinin Combination Therapy (ACT) was therefore subsequently introduced in 2001 [9]. The use of a partner drug with a prolonged half-life was aiming at clearing the remaining parasites after artemisinin action and reducing the chances of parasites surviving and developing resistance against the drug [10].

ACT treatment failures attributed to artemisinin resistance were recently reported in SEA especially in Thailand-Cambodia border and Myanmar [9,11,12]. It has been reported that some mutations in the Kelch 13 region (K13) of Plasmodium falciparum genome are responsible for the development of artemisinin resistance [11]. Since the efficacy of each ACT is dependent on the performance of artemisinin and its partners drugs [10], we carried out a systematic review and network meta-analysis (SR/NMA) with the aim of understanding the effectiveness of the current ACTs in relation to recrudescence, reinfection, parasitological failure, and clinical failure in the treatment of patients with malaria. We also aimed to identify the current status of artemisinin resistance in relation to its geographical distribution and artemisinin molecular markers.

\section{Methods}

\subsection{Study protocol registration}

Our study conformed to the Preferred Reporting Items for Systematic Review and Meta-Analysis (PRISMA) guideline [13] and the PRISMA checklist is presented in Supplementary Table S1. The study protocol was developed and registered in PROSPERO under number CRD42018087574.

\subsection{Search strategy and study selection}

Electronic search was conducted without language restrictions to identify relevant articles published from 2001 to 2017 in several databases including; PubMed, Scopus, Web of Science (ISI), Google Scholar, EMBASE, Virtual Health Library (VHL), Global Health Library (GHL), Cochrane, Population Information Online (POPLINE), New York Academy of Medicine Grey Literature Report (NYAM), and System for Information on Grey Literature in Europe (SIGLE). Our search term: (artemisinin OR qinghaosu OR artemisininum OR dihydroartemisinin OR artelinic acid OR artenimol OR artemotil OR artesunate OR artemether OR coartem) AND (resistance OR resist OR response OR respond OR recurrence $O R$ recurrent $O R$ recrudescence OR relapse OR persistent OR efficacy) AND (patients or patient). More details about the search strategy for other databases can be found at (Supplementary Table S2). The search was supplemented with manual searches for further relevant articles by reviewing the references of the key included papers, checking for similar articles and articles that cited the key papers on Google Scholar and PubMed [14].

We included articles that reported artemisinin resistance based on WHO definition of confirmed or suspected endemic artemisinin resistance. For the NMA, we included articles that reported treatment failures in the form of recrudescence of Plasmodium falciparum within 28 days after treatment. We excluded in vitro studies, reviews, poster presentations and studies with no accessible or extractable data. Potential eligible studies were reviewed and selected independently by three reviewers based on the selection criteria. Any discrepancies in selection were resolved by consensus.

\subsection{Data extraction and quality assessment}

Data extraction was conducted by three independent reviewers using a standardized extraction sheet that included basic information about the studies such as title, author names, year of publication, location of the study, sample size, dosage, and regimen. The specific information included half-life of parasite clearance slope, day three parasitemia, clinical and treatment failures, recrudescence, reinfection, and genetic mutations of Plasmodium falciparum parasites. Quality assessment was performed using ROB Cochrane tool for randomized controlled trials (RCTs) and National Institute of Health (NIH) tool for cross-sectional studies [15]. Any discrepancies regarding extracted data and quality assessment were resolved by consensus.

\subsection{Definition of artemisinin resistance and treatment failure used from WHO}

\subsubsection{Artemisinin resistance}

Confirmed endemic artemisinin resistance is defined as the presence of $>5 \%$ of patients carrying any of the $\mathrm{K} 13$ resistance-confirmed mutations, all of whom have been found to have either persistent parasitemia by microscopy on day three or a half-life of the parasite clearance slope of $>5 \mathrm{~h}$ after treatment. Suspected endemic artemisinin resistance is defined as the presence of $>10 \%$ of patients with a halflife of the parasite clearance slope of $>5 \mathrm{~h}$ after treatment with ACT or artesunate (AS) alone; or $>5 \%$ of patients carrying K13 resistanceconfirmed mutations; or $>10 \%$ of patients with persistent parasitemia by microscopy at $72 \mathrm{~h} \mathrm{[16].}$

\subsubsection{Treatment failure}

Inability to clear malarial parasitemia or resolve clinical symptoms despite administration of an antimalarial medicine [17].

\subsubsection{Slope half-life}

Time needed for parasitemia to be reduced by half [18].

\subsection{Data analysis}

Network meta-analysis (NMA) was performed under a frequentist framework using binomial likelihood for dichotomous outcomes. Conventional pairwise meta-analysis (MA) was performed whenever NMA was not feasible. Effect sizes were synthesized using fixed-effect model with Mantel-Haenszel method except when there was a significant heterogeneity, for which random-effects model with Der Simonian and Laird method was used. Statistical heterogeneity was assessed based on the degree of heterogeneity variance $(\tau 2)$. I-square $\left(I^{2}\right)$ test was used to measure inconsistency. Heterogeneity was 
considered significant if $P$-value of $\mathrm{Q}$ statistic was $<0.10$ and/or $I^{2}$ was $>50 \%$ [19], with the assumption that all treatment comparisons having the same degree of heterogeneity. Outcomes were ranked based on interpretation of the P-scores which represent the probability of superiority of any given treatment over the others, as a similar estimate of the surface under the cumulative ranking area (SUCRA) [17]. The NMA was reported in accordance with the modified PRISMA guidelines for network meta-analyses [20]. Dichotomous data was presented as relative risk (RR), with 95\% confidence interval (CI). All analyses were performed in R 3.2.3 [21-23] using the 'meta', and 'netmeta' packages [24]. A subgroup analysis of studies published after 2012, when the molecular marker of resistance was identified [25] was also conducted to elucidate the current trend of overall performance of ACTs.

\section{Results}

\subsection{Literature search and study characteristics}

The electronic database search yielded 8400 studies with a total of 260 articles included for full-text review. Additional 6 studies identified from manual search were also included. A total of 82 studies were eligible for data extraction, and all of which were included for qualitative and quantitative analysis (Fig. 1). The mean age ranged between 0.07 and 44.1 years old, with males constituting a slightly higher proportion than females $(10,341=51.4 \%)$ The follow-up point was at 28 days. Detailed patient characteristics of included studies are shown in Supplementary Table S3.

In total, 17 different ACTs were included with artemether-lumefantrine (AL) being the most commonly used ACT in 34 studies and
Table 1

Table of drug abbreviations.

\begin{tabular}{ll}
\hline Abbreviation & Artemisinin-based combination therapies \\
\hline AL & Artemether-Lumefantrine \\
AL + PQ & Artemether-Lumefantrine-Primaquine \\
ART + PIP & Artemisinin-Piperaquine \\
AS & Artesunate \\
AS + AQ & Artesunate-Amodiaquine \\
AS + ATPG & Artesunate-Atovaquone-Proguanil \\
AS + CD & Artesunate-Chlorproguanil-Dapsone \\
AS + CQ & Artesunate-Chloroquine \\
AS + MQ & Artesunate-Mefloquine \\
AS + NQ & Artemisinin-Naphthoquine \\
AS + PYN & Artesunate-Pyronaridine \\
AS + SMP & Artesunate-Sulphamethoxy-Pyrazine/Pyrimethamine \\
AS + SP & Artesunate-Sulphadoxine-Pyrimethamine \\
DHA + MQ & Dihydroartemisinin-Mefloquine \\
DHA + PIP & Dihydroartemisinin-Piperaquine \\
AS + AZ & Artesunate-Azithromycin \\
DHA + PQP + TM & Dihydroartemisinin-Piperaquine-Trimethoprim \\
\hline
\end{tabular}

treatment failure reported in 32 of the 34 studies. Table 1 shows the drugs abbreviation used.

\subsection{Risk of bias assessment}

The quality assessment was performed for 72 RCTs, 8 cross-sectional studies and 2 prospective cohort studies. Risk of bias assessment is illustrated in Fig. 2 and Supplementary Fig. S1.

Most of the studies had low risk of bias especially for reporting bias $[26,27]$. The highest bias was observed in allocation of drugs where

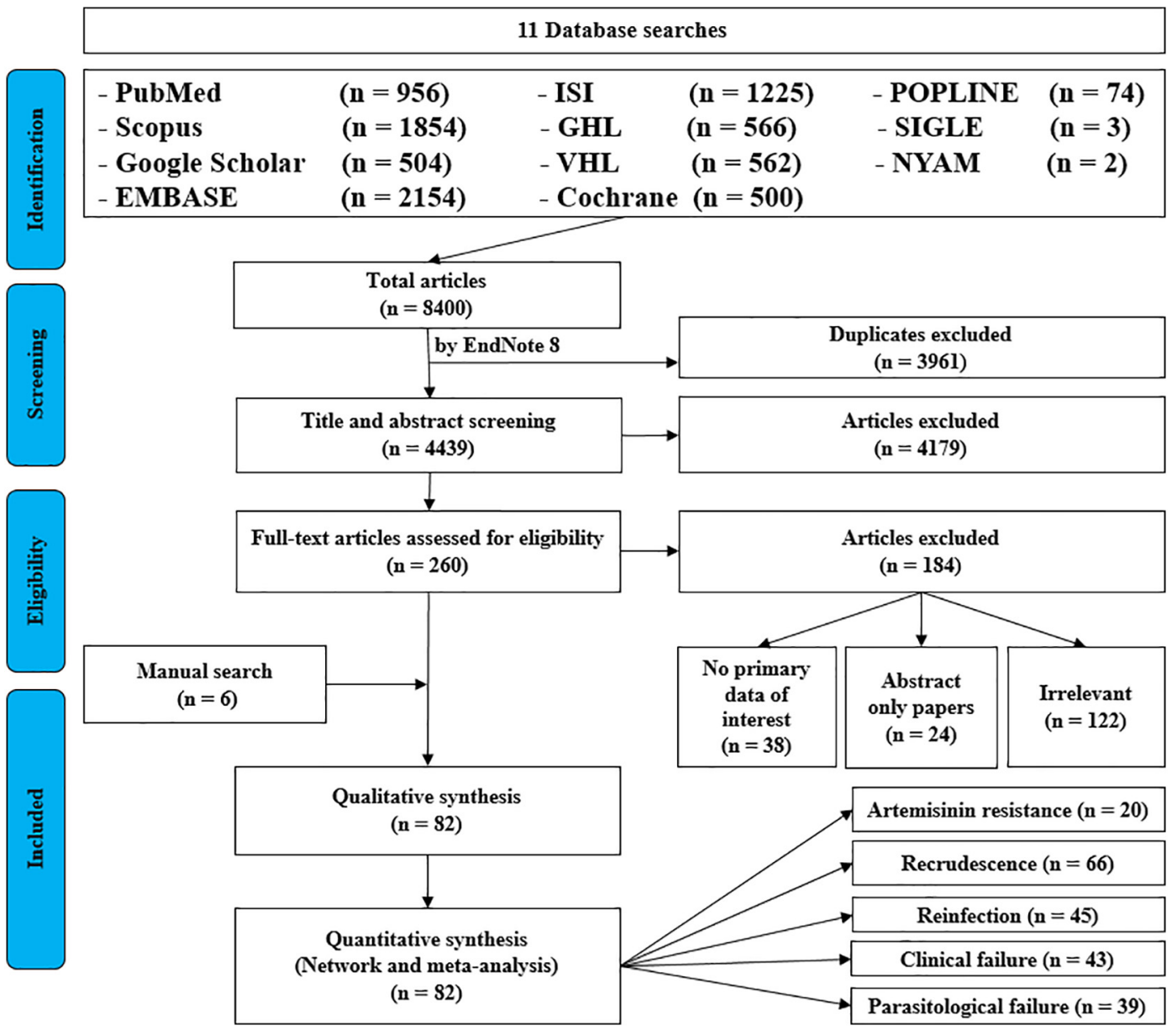

Fig. 1. PRISMA flow diagram of studies' screening and selection. 


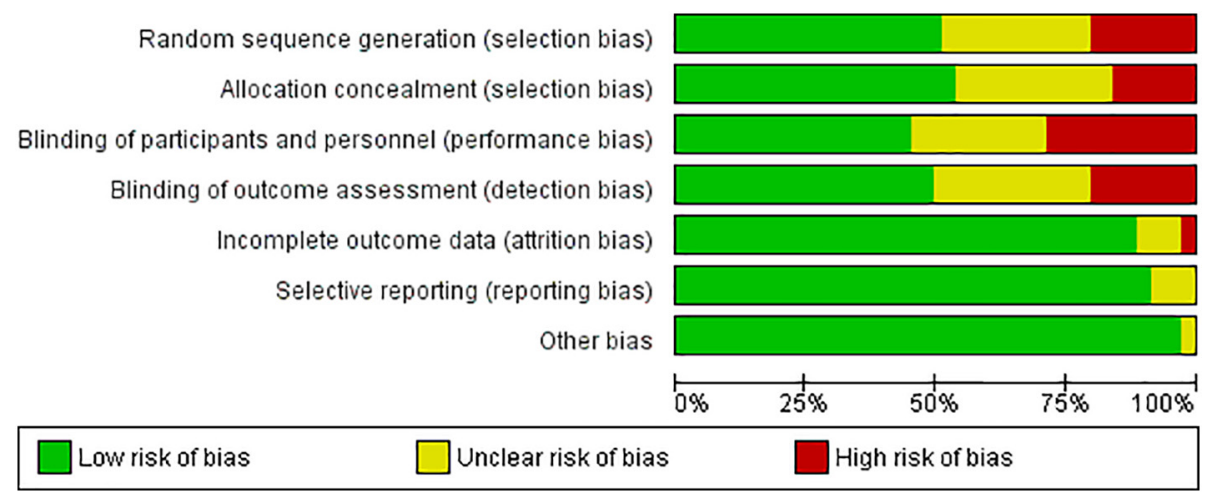

Fig. 2. Risk of bias assessment graph.

there was no blinding of patients to the drugs given [28,29]. This might be due to the difficulty of blinding in single arm-studies where only one drug was used. Although many studies had well-defined inclusion criteria for patient recruitment, many of them did not make it clear on the criteria used in the randomization process for inclusion $[29,30]$. In summary, most included RCTs were rated to be of either moderate or high quality. As for cross sectional and cohort studies, the risk of bias was minimal although many studies did not mention the justification of the sample size used in the study (Fig. 2).

\subsection{Artemisinin resistance}

Out of the 20 studies that reported suspected artemisinin resistance, 6 studies reported confirmed artemisinin resistance, while 14 studies reported suspected resistance. Apart from a few studies, many of included studies investigated all the measure for resistance where by, 11 studies reported half parasite clearance slope of $>5 \mathrm{~h}$ for $>10 \%$ of the patients included per protocol. Ten studies reported day three parasitemia for $>10 \%$ of the patients recorded, and 11 studies reported presence of confirmed $\mathrm{K} 13$ mutation in $>5 \%$ of the patients recorded as per protocol (Supplementary Table S4; Table 1). A map generated showing the locations of the studies that reported suspected and confirmed resistance are shown in Supplementary Fig. S2 with blue color showing the regions with suspected resistance while Magenta color showing regions with confirmed resistance as reported in the study.

K13 mutations associated with artemisinin resistance were reported in Cambodia, Thailand, Myanmar, Vietnam, and Laos with none reported outside SEA region including Africa. [25,31]. K13 C580Y was the most abundant $\mathrm{K} 13$ mutation associated with resistance, comprising $63.1 \%$ of all reported $\mathrm{K} 13$ mutations associated with resistance $(n=693)$ and $42.5 \%(n=1025)$ of all K13 mutations including nonsynonymous mutations reported. F446I and R539T had $11.0 \%$ and

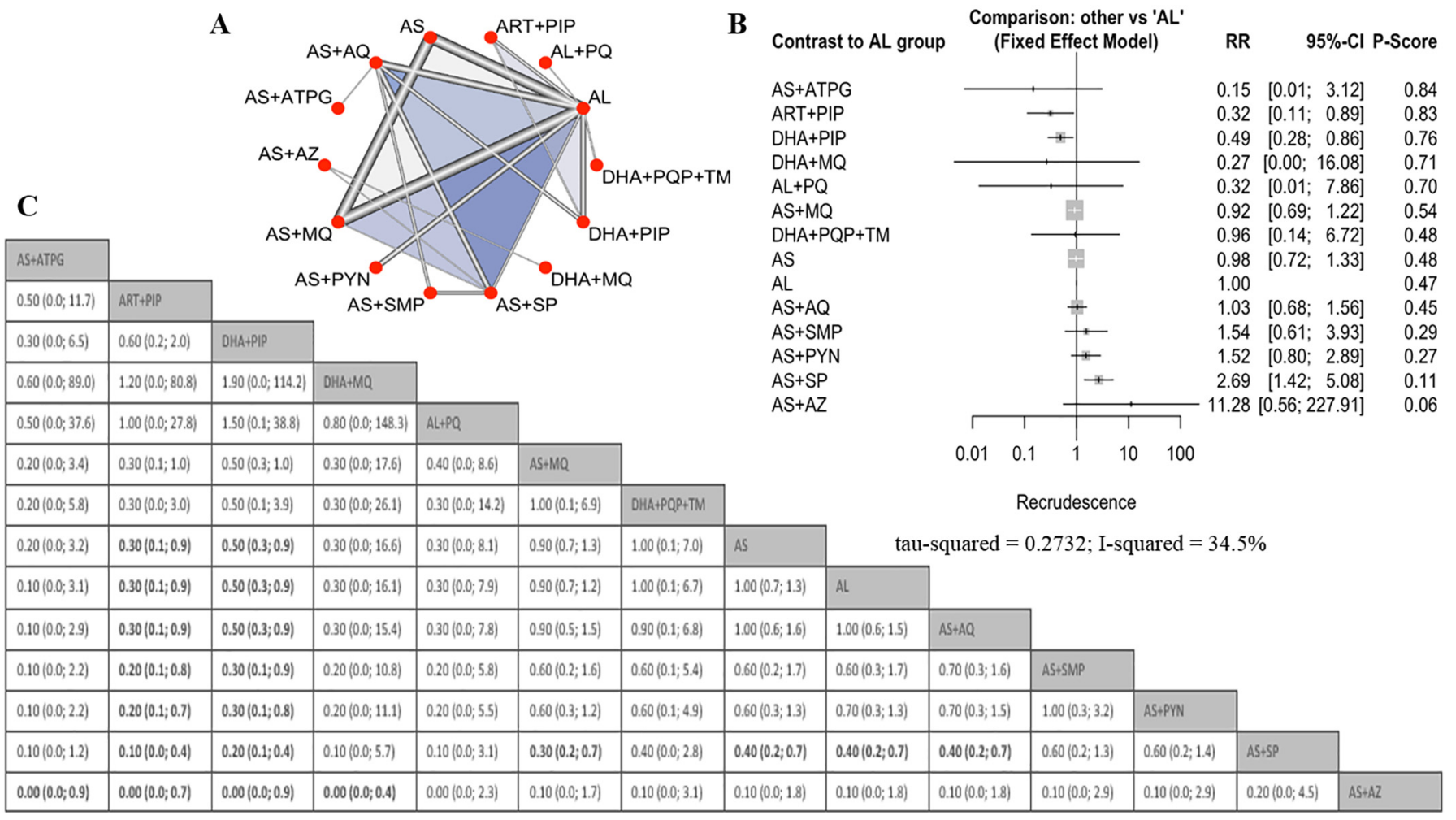

Fig. 3. Network meta-analysis results of recrudescence for all artemisinin-based combination therapies. (A) Network graph showing the comparison between the drugs, each node represents one ACT used, the edge represents a drug trial and thickness of the edge represents the number of trials between the drugs. (B) A forest plot generated by comparing the ACT with artemether-lumefantrine. (C) The league table represents the network meta-analysis estimates, with ACT on the top left having the lowest Risk ratio. 
$18.2 \%$ of the mutation associated with resistance respectively while $\mathrm{Y} 493 \mathrm{H}, \mathrm{I543T}, \mathrm{P} 574 \mathrm{~L}$ and P553L had an abundance of $4.3 \%, 0.9 \%$, $0.9 \%$ and $1.7 \%$ respectively. No new mutation (non-synonymous mutation) was shown to be associated with artemisinin resistance. Supplementary Table S5 shows the countries where each of the K13 mutation associated with artemisinin resistance was reported, the locations and year of the study. C580Y and F446I were the two most commonly K13 mutations reported with six studies each.

\subsection{Treatment failure}

We analyzed our outcomes - recrudescence, reinfection, clinical failure, parasitological failure - by using traditional MA and NMA. Firstly, MA was done for all included studies from 2001 to 2017 by grouping the studies according to the treatment used (Supplementary Figs. S3-S6).

According to the analysis of treatment groups, there was a significant increase in recrudescence cases when AS was used alone $[95 \%$ confidence intervals (CI)] (0.27 [0.15; 0.42]) (Supplementary Fig. S3). In addition, AS led to a significantly higher proportion of reinfection (0.22 $\quad[0.14 ; 0.32])$ whereas dihydroartemisinin-piperaquine (DHA + PIP) resulted in a significantly lower proportion of reinfection (0.04 [0.02; 0.09]) (Supplementary Fig. S4). The number of cases of clinical failure was found to have a significant increase when AS in combination with sulphadoxine-pyrimethamine (AS +SP), chlorproguanil-dapsone $(\mathrm{AS}+\mathrm{CD})$ or with piperaquine (ART + PIP) were used (0.12 [0.07; 0.19]), (0.17 [0.14; 0.22], (0.12 [0.07; 0.19], respectively) (Supplementary Fig. S5). Drug combinations (dihydroartemisinin-piperaquine-trimethoprim) (DHA + PQP + TM) and (artesunate-pyronaridine) (AS + PYN) showed a significant decrease in parasitological failure rate when used for treatment $(0.01[0.00 ; 0.04])$ and $(0.01$ [0.01; 0.02], respectively). However, there was a significant increase of the same when artesunate-azithromycin $(\mathrm{AS}+\mathrm{AZ})$ and $\mathrm{AS}+\mathrm{CD}$ were used $(0.27[0.18 ; 0.39])$ and $(0.18[0.15,0.22]$, respectively)
(Supplementary Fig. S6).

Following these results, we carried out pairwise NMA to compare all ACTs together with $\mathrm{AL}$ as the reference points since it was the most widely used ACT in the included studies (Figs. 3-6). From all ACTs used, DHA + PIP was found to have the highest efficacy with the lowest RRs in reinfection, clinical failure, and parasitological failure, while third in recrudescence when compared to the other drugs (RR, 95\% CI, P-score) ( 0.29 [0.16; 0.52], $P=.98),(0.23$ [0.16; 0.32], $P=.85)$, (0.51 [0.28; 0.93], $P=.93$ ), and (0.49 [0.28; 0.86], $P=.76)$, respectively). AS + AZ was found to be the least effective drug with the highest RRs in recrudescence, reinfection, and parasitological failure ((11.28 [0.56; 227.91], $P=.06$ ), (10.53 [0.49; 226.27], $P=.07$ ), (32.58 [1.34; 793.93], $P=.97$ ) (Figs. 3-6).

In order to elucidate the current trend of overall performance of ACT in post-resistance era, when the molecular marker of resistance was identified, a subgroup analysis of studies published after 2013 was also carried out. There was a high proportion of recrudescence with DHA + PIP especially in Cambodia (RR, 95\% CI, (0.07 [0.04;0.10]) and $(0.20[0.15 ; 0.26]))$ respectively (Fig. 7) with clinical failure being slightly elevated as compared to the previous analysis (Random effects model, RR, 95\% CI (0.03 [0.01;0.08])) (Fig. 8) versus (Random effects model, RR, 95\% CI (0.03 [0.02;0.05])) (Supplementary Fig. S5). Analysis on the Vietnam studies reported many cases of day three parasitemia and confirmed K13 mutation following the usage of the DHA + PIP (Supplementary Table S4). There is no drug combination that performed better than the others in relation to treatment failure as opposed to the first analysis. With many of the recent studies focusing on the efficacy and resistance of the DHA + PIP, there were no studies comparing it with other drugs, hence none was included in the NMA, which showed no significant differences between the ACTs used (Fig. 9).

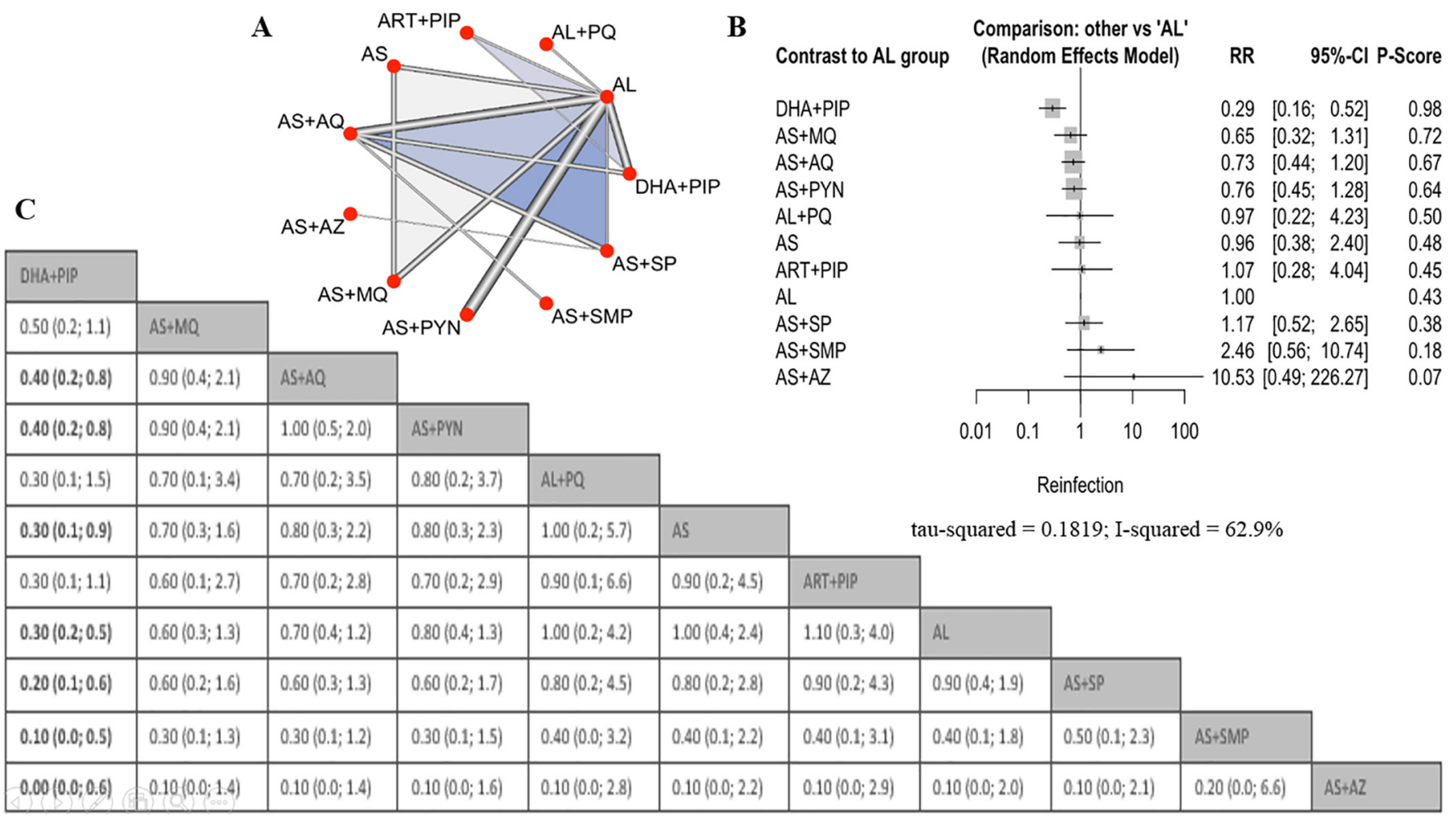

Fig. 4. Network meta-analysis results of reinfection for all artemisinin-based combination therapies. (A) Network graph of reinfection with majority of the drug being compared to AL while AS + AZ having few drug trials comparisons. (B) Dihydroartemisinin-piperaquine showing significant reduction in reinfection as compared to other drugs. This is also reflected in the league table (C). 


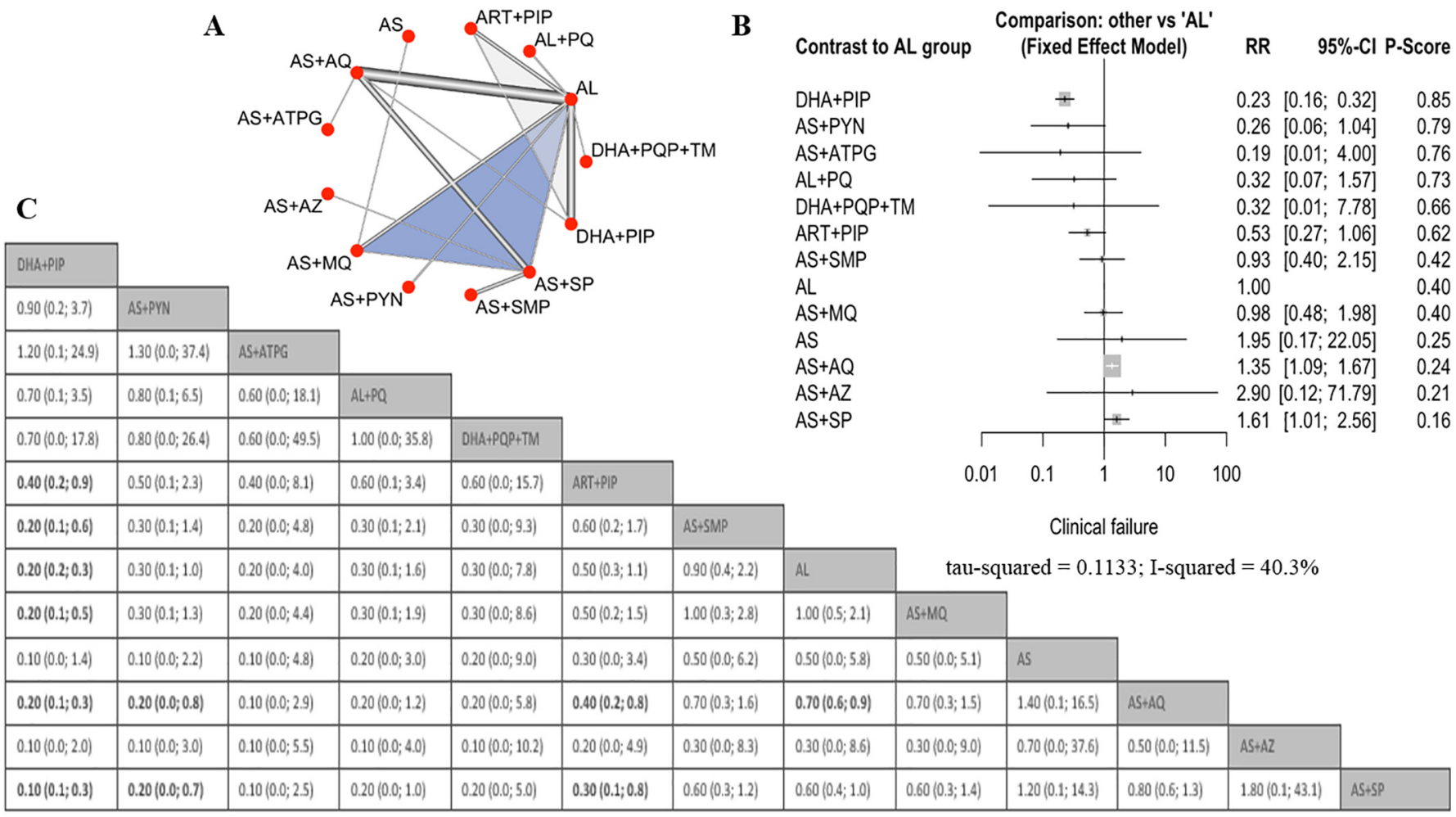

Fig. 5. Network meta-analysis results of clinical failure for all artemisinin-based combination therapies showing the different combination of drugs used and the comparison made between them, the drug AS is shown to have few comparisons as opposed to the other ACTs (A). DHA + PIP was shown to have high a significant difference in reducing the cases (B), and it was also shown to have the lowest risk ratio in league table (C).

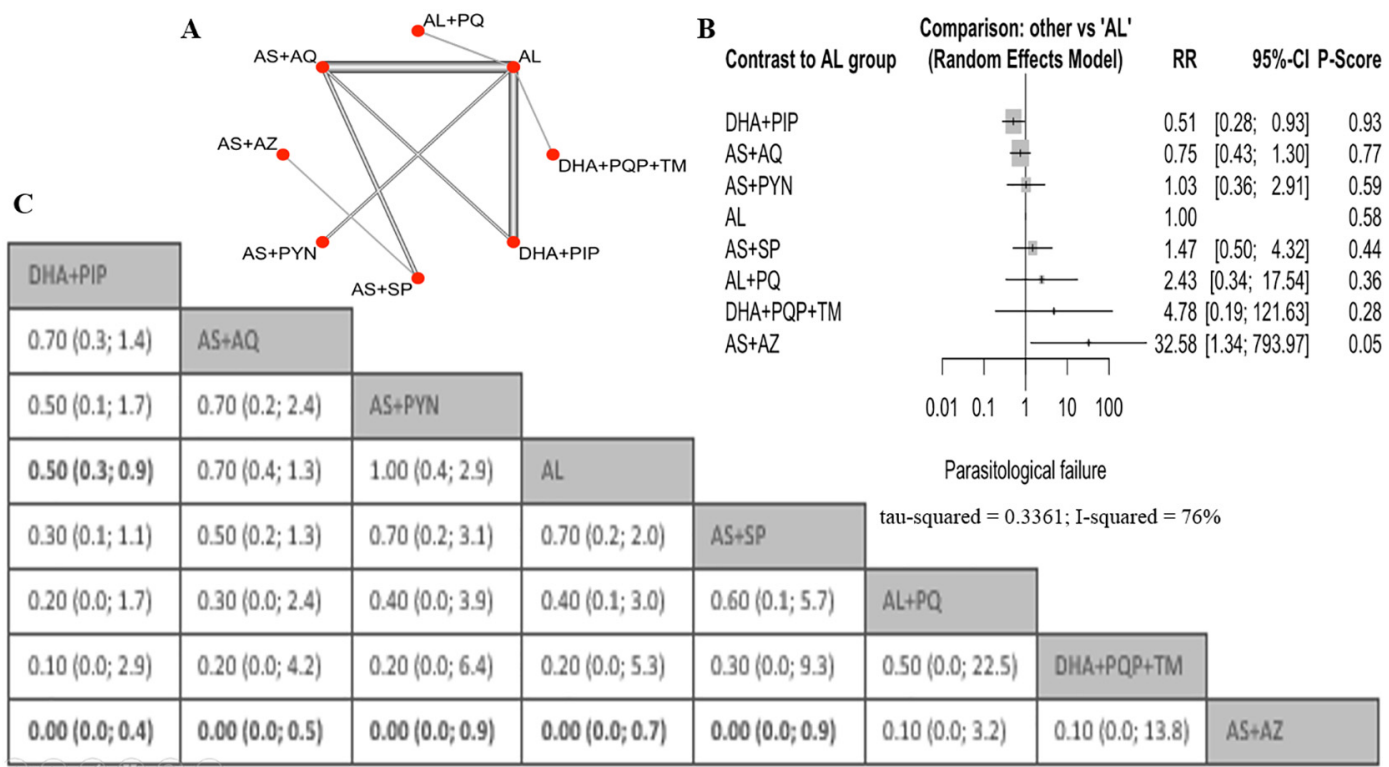

Fig. 6. Network meta-analysis results of parasitological failure for all artemisinin-based combination therapies. Few drug trials included as shown in (A) but DHA + PIP is shown to have the lowest risk ratio in (B) and (C).

\section{Discussion}

In this study, we highlighted the geographical distribution and mutations associated with artemisinin resistance. This was followed by a comprehensive comparison between different ACTs in terms of treatment failure, recrudescence, and reinfection. A subgroup analysis was also performed to elucidate the current trend of overall performance of ACTs since 2013. The overall NMA showed that artemisinin monotherapy has a high risk of recrudescence. We believe that this may be a result of its rapid mode of action and a short half-life leading to its inability to clear all the malaria parasites in the body [8]. The same is observed for chlorproguanil-dapsone in which rapid elimination from the body is believed to be the reason for treatment failure [32]. Sulfadoxine-pyrimethamine (SP) revealed a high risk of clinical failure, which may be a result of Plasmodium falciparum resistance against the drug. However, its prolonged half-life has been shown to protect against new cases of reinfection [15], which is in line with our findings.

DHA + PIP has been adopted in several countries in SEA following 


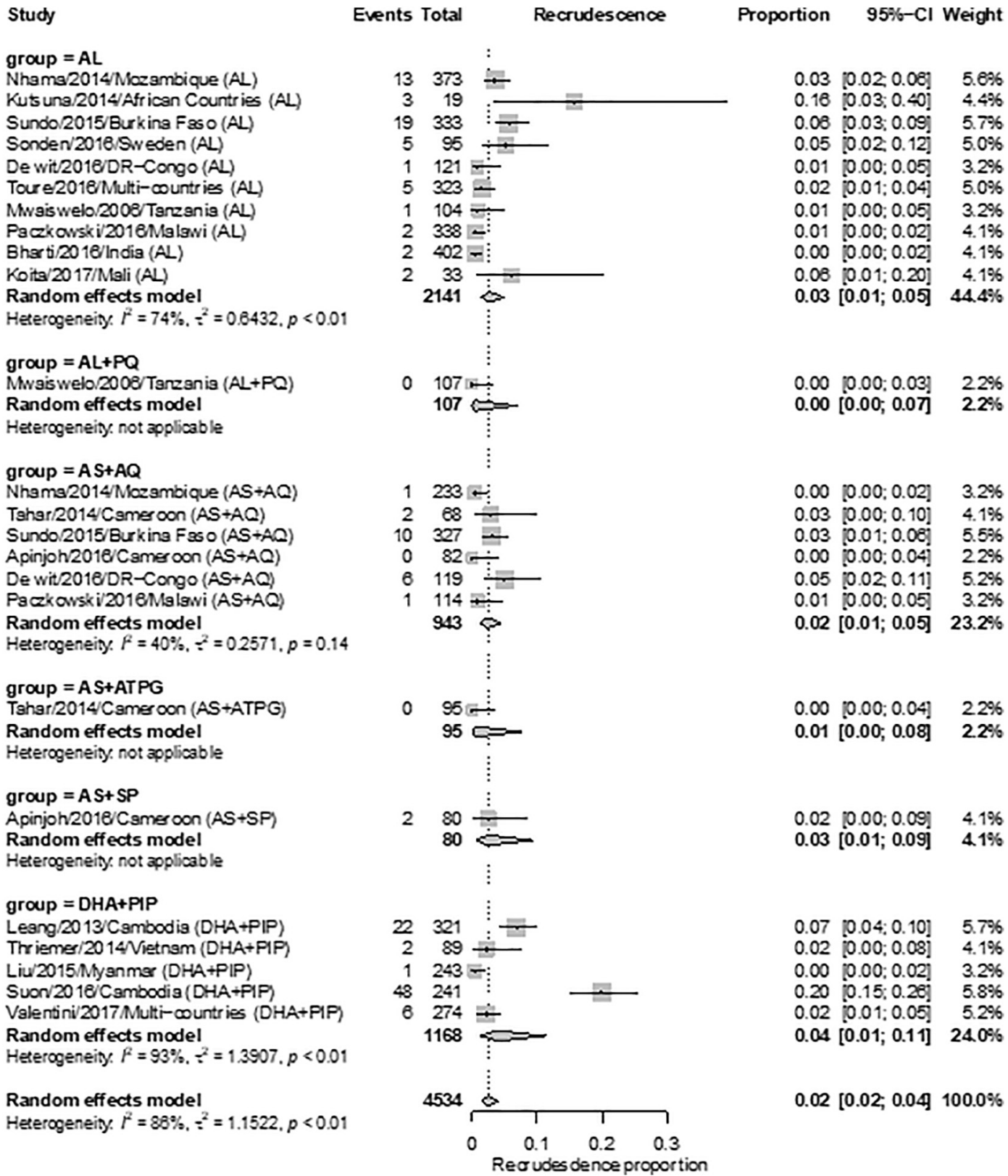

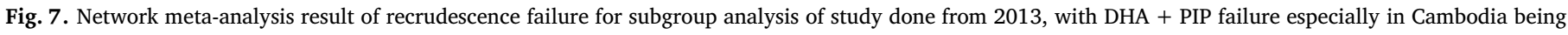
high.

its high efficacy against malaria parasites in earlier years. Although our overall NMA showed that DHA + PIP outperforms other ACTs, this was not the case in the subgroup analysis of recent studies. The subgroup analysis has found no significant difference in recrudescence cases, clinical failure and parasitological failure between different ACTs, hence indicating poor efficacy and resistance against the drugs. The partial protection against the reinfection cases by the drug is believed to be associated with PIP prolonged half-life in the body [33,34]. DHA + PIP performance in some counties like Cambodia was shown to have decreased in 2013, 3 years after it was adapted as the first line of treatment [35]. This is believed to be associated with prior exposure of the drug PIP before its adoption in the country [33,34]. In addition, in vitro studies from the included studies have shown that PIP survival assay and ring stage survival assay in artemisinin resistance region like Pailin has increased to up to $6 \mathrm{~h}$ [36] which seems to give more evidence of resistance against the drug. Similar trend has been observed in Vietnam where the drug was adopted in 2008 [36] leading to a consideration for reverting to AS + MQ in view of the rising treatment failure and resistance cases associated with DHA + PIP in the recent years [37].

Some of the studies conducted in SEA suggest that failure of different ACTs in the region is as a result of artemisinin failure, which leads to overburdening of the partner drug and subsequent failure of the combined therapy [38]. However, there are other studies that suggest that the failure of the ACTs is as a result of both artemisinin resistance and partner drug failure in the region it [35,37].

Following the failure of all ACTs against resistant parasites, several studies on triple ACT are currently underway in order to identify an effective combination of 3 antimalarial drugs to be used in SEA. A study registered in ClinicalTrials.gov Identifier: NCT03355664 [32] for example, is working on triple ACT AL + AQ with the ACT AL in Cambodia and Vietnam (TACT-CV).

Our study has shown that artemisinin resistance has only been reported in SEA even though several randomized controlled trials have 


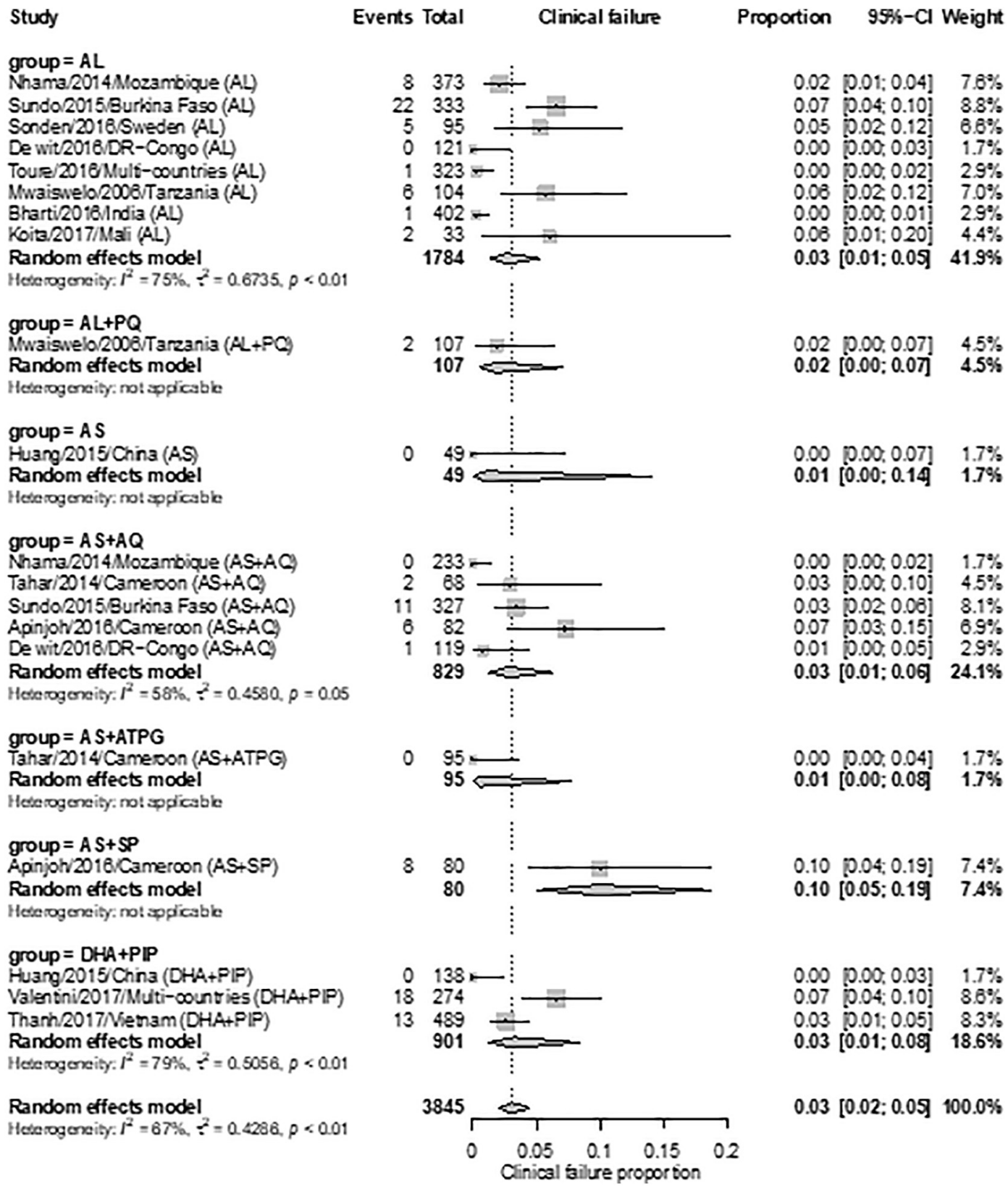

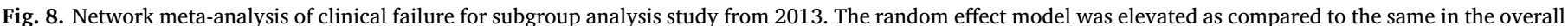
analysis.

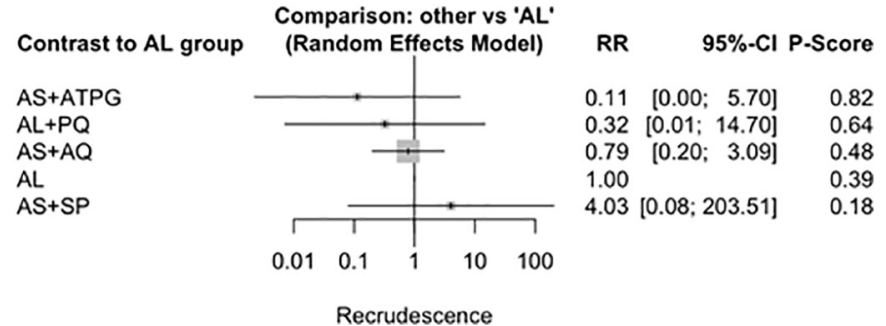

Fig. 9. Pairwise network meta-analysis of recrudescence for subgroup analysis study from 2013, there was no significant difference between the drugs.

been carried out in Africa in attempt to identify K13 mutation associated with artemisinin resistance [11,31]. Recent studies in Tanzania and Democratic Republic of Congo have not found evidence of artemisinin resistance $[39,40]$. However, a recent publication of ex vivo studies conducted in Uganda reported to have found K13 mutation that can be associated with artemisinin resistance [41].

Geographical mapping of the study sites with reported resistance has shown that the resistance is mostly confined to small regions within the countries, where the emergence of resistance may be linked to the genetic factors in a given parasite population [42,43].

K13 mutations were reported in certain geographical locations with I543T, Y493H and P553L being reported only in Vietnam, while F446I was only found in Myanmar and China [27,44,45]. These findings are similar to a study with focus on mapping of the K13 mutations [42]. C580Y was the most reported K13 mutation that was found in almost all the countries apart from China [44,36,46,47]. As for the K13 R539T, it has been reported in four countries, namely Laos, Vietnam, Thailand and Cambodia [44,46,48]. 


\subsection{Limitations}

Our study considered studies with only standardized dosage while studies comparing different dosages were not included. This led to the inclusion of some ACTs with small sample size that might have led to the high heterogeneity for reinfection and parasitological failure. To overcome this, we carried out pairwise NMA of the ACTs, to compare the results and magnify the ACTs with small samples. We also assumed that all the dosage given were fully absorbed by the body as we were unable to account for the drug bioavailability, which is affected by pharmacokinetics and pharmacodynamics of each drug.

\subsection{Conclusion}

With different K13 mutations in SEA leading to ACT resistance, more surveillance studies for artemisinin resistance and K13 mutations need to be carried out. It is imperative that a new drug or formulation against artemisinin resistance is developed before further spread of resistance.

\section{Declarations}

\section{Ethics approval and consent to participate}

Not applicable.

\section{Consent for publication}

Not applicable.

\section{Availability of data and material}

The datasets used and/or analyzed during the current study are available from the corresponding author on reasonable request.

\section{Author contributions}

NTH and KH were responsible for the idea and supervision. Screening and extraction were done by all authors under the supervision of NTH and KH. Data analysis and its interpretation were done by NLV, PGM, SKL, GMT and NTH. Tables and figures were prepared by GMT and SKL. All authors contributed to the manuscript writing and approved the final version.

\section{Competing interest}

None of the authors has any competing interest to declare.

\section{Funding}

This study was conducted (in part) at the Joint Usage/Research Center on Tropical Disease, Institute of Tropical Medicine, Nagasaki University, Japan. The funders had no role in the study design, data collection and analysis, decision to publish or preparation of the manuscript.

Supplementary data to this article can be found online at https:// doi.org/10.1016/j.parint.2019.04.016.

\section{Acknowledgments}

To Dr. Tran Tinh Hien (Oxford University Clinical Research Unit, Welcome Trust Major Overseas Program, Ho Chi Minh City, Vietnam) for constructive critic and guidance in the work.

\section{References}

[1] W.F. Sheet, World Malaria Report 2015, Back to cited text, 1 (2016), pp. 87-92.

[2] R.L. Nevin, A.M. Croft, Psychiatric effects of malaria and anti-malarial drugs: historical and modern perspectives, Malar. J. 15 (1) (2016) 332.

[3] U. D'Alessandro, H. Buttiens, History and importance of antimalarial drug resistance, Tropical Med. Int. Health 6 (11) (2001) 845-848.

[4] T.E. Wellems, Plasmodium chloroquine resistance and the search for a replacement antimalarial drug, Science 298 (5591) (2002) 124-126.

[5] C. Wongsrichanalai, et al., Epidemiology of drug-resistant malaria, Lancet Infect. Dis. 2 (4) (2002) 209-218.

[6] X.Z. Su, L.H. Miller, The discovery of artemisinin and the Nobel Prize in physiology or medicine, Sci. China Life Sci. 58 (11) (2015) 1175-1179.

[7] N.J. White, Malaria parasite clearance, Malar. J. 16 (1) (2017) 88.

[8] L.A. Walker, D.J. Sullivan Jr., Impact of extended duration of artesunate treatment on parasitological outcome in a cytocidal murine malaria model, Antimicrob. Agents Chemother. 61 (4) (2017).

[9] Q. Cheng, D.E. Kyle, M.L. Gatton, Artemisinin resistance in Plasmodium falciparum: a process linked to dormancy? Int. J. Parasitol. Drugs Drug Resist. 2 (2012) 249-255.

[10] N.D. Alexander, Rationale and prospects of combination therapy as a strategy for delaying antimalarial drug resistance, P N G Med J 44 (1-2) (2001) 1-5.

[11] E.A. Ashley, et al., Spread of artemisinin resistance in Plasmodium falciparum malaria, N. Engl. J. Med. 371 (5) (2014) 411-423.

[12] A. Bhumiratana, et al, Border malaria associated with multidrug resistance on Thailand-Myanmar and Thailand-Cambodia borders: transmission dynamic, vulnerability, and surveillance, Biomed. Res. Int. 2013 (2013) 363417.

[13] L. Shamseer, et al., Preferred reporting items for systematic review and meta-analysis protocols (PRISMA-P) 2015: elaboration and explanation, BMJ 349 (2015) g7647.

[14] M. Vassar, P. Atakpo, M.J. Kash, Manual search approaches used by systematic reviewers in dermatology, J. Med. Libr. Assoc. 104 (4) (2016) 302.

[15] M.L. Gatton, L.B. Martin, Q. Cheng, Evolution of resistance to sulfadoxine-pyr imethamine in Plasmodium falciparum, Antimicrob. Agents Chemother. 48 (6) (2004) 2116-2123.

[16] Organization, W.H, Artemisinin and Artemisinin-Based Combination Therapy Resistance: Status Report, World Health Organization, 2017.

[17] Organization, W.H, Global Report on Antimalarial Drug Efficacy and Drug Resistance: 2000-2010, (2010).

[18] N. White, The parasite clearance curve, Malar. J. 10 (1) (2011) 278.

[19] J. Higgins, S. Green, Cochrane Handbook for Systematic Reviews of Interventions Version 5.1. 0, The Cochrane Collaboration, 2011 Confidence Intervals.

[20] B. Hutton, et al., The PRISMA extension statement for reporting of systematic reviews incorporating network meta-analyses of health care interventions: checklist and explanations, Ann. Intern. Med. 162 (11) (2015) 777-784.

[21] R.C. Team, R: A Language and Environment for Statistical Computing, vol. 2015, R Foundation for Statistical Computing, 2014.

[22] R.C. Team, R: A Language and Environment for Statistical Computing, [Internet] R Foundation for Statistical Computing, Vienna, Austria, 2015 Document freely available on the internet at http://www.r-project.org.

[23] G. Schwarzer, Meta: an R package for meta-analysis, R news 7 (3) (2007) 40-45.

[24] G. Rücker, et al., Netmeta: Network Meta-Analysis Using Frequentist Methods, R Package Version 0.8-0 (2015).

[25] R. Amato, et al., Genetic markers associated with dihydroartemisinin-piperaquine failure in Plasmodium falciparum malaria in Cambodia: a genotype-phenotype association study, Lancet Infect. Dis. 17 (2) (2017) 164-173.

[26] A.K. Tshefu, et al., Efficacy and safety of a fixed-dose oral combination of pyronaridine-artesunate compared with artemether-lumefantrine in children and adults with uncomplicated Plasmodium falciparum malaria: a randomised non-inferiority trial, Lancet 375 (9724) (2010) 1457-1467.

[27] Z. Wang, et al., Artemisinin resistance at the China-Myanmar border and association with mutations in the K13 propeller gene, Antimicrob. Agents Chemother. 59 (11) (2015) 6952-6959.

[28] N.G. Schwarz, et al., 5-day non-observed artesunate monotherapy for treating uncomplicated falciparum malaria in young Gabonese children, Am. J. Trop. Med. Hyg. 73 (4) (2005) 705-709.

[29] H. Liu, et al., In vivo monitoring of dihydroartemisinin-piperaquine sensitivity in Plasmodium falciparum along the China-Myanmar border of Yunnan Province, China from 2007 to 2013, Malar. J. 14 (1) (2015) 47.

[30] K.M. Tun, et al., Parasite clearance rates in upper Myanmar indicate a distinctive artemisinin resistance phenotype: a therapeutic efficacy study, Malar. J. 15 (1) (2016) 185.

[31] M. Ocan, et al., Prevalence of K13-propeller gene polymorphisms among Plasmodium falciparum parasites isolated from adult symptomatic patients in northern Uganda, BMC Infect. Dis. 16 (1) (2016) 428.

[32] P. Winstanley, Chlorproguanil-dapsone (LAPDAP) for uncomplicated falciparum malaria, Tropical Med. Int. Health 6 (11) (2001) 952-954.

[33] R. Leang, et al., Efficacy of dihydroartemisinin-piperaquine for treatment of uncomplicated Plasmodium falciparum and Plasmodium vivax in Cambodia, 2008 to 2010, Antimicrob. Agents Chemother. 57 (2) (2013) 818-826.

[34] S. Yeung, et al., Antimalarial drug resistance, artemisinin-based combination therapy, and the contribution of modeling to elucidating policy choices, Am. J. Trop. Med. Hyg. 71 (2_suppl) (2004) 179-186.

[35] D.L. Saunders, P. Vanachayangkul, C. Lon, Dihydroartemisinin-piperaquine failure in Cambodia, N. Engl. J. Med. 371 (5) (2014) 484-485.

[36] N.V. Thanh, et al., Rapid decline in the susceptibility of Plasmodium falciparum to 
dihydroartemisinin-piperaquine in the south of Vietnam, Malar. J. 16 (1) (2017) 27.

[37] B.Q. Phuc, et al., Treatment failure of dihydroartemisinin/piperaquine for Plasmodium falciparum malaria, Vietnam, Emerg. Infect. Dis. 23 (4) (2017) 715.

[38] C. Amaratunga, et al., Dihydroartemisinin-piperaquine resistance in Plasmodium falciparum malaria in Cambodia: a multisite prospective cohort study, Lancet Infect. Dis. 16 (3) (2016) 357-365.

[39] M.A. Kakolwa, et al., Efficacy and safety of artemisinin-based combination therapy, and molecular markers for artemisinin and piperaquine resistance in mainland Tanzania, Malar. J. 17 (1) (2018) 369.

[40] P.I. Mayengue, et al., No polymorphisms in K13-propeller gene associated with artemisinin resistance in Plasmodium falciparum isolated from Brazzaville, republic of Congo, BMC Infect. Dis. 18 (1) (2018) 538.

[41] M. Ikeda, et al., Artemisinin-resistant Plasmodium falciparum with high survival rates, Uganda, 2014-2016, Emerg. Infect. Dis. 24 (4) (2018) 718-726.

[42] D. Menard, et al., A worldwide map of Plasmodium falciparum K13-propeller polymorphisms, N. Engl. J. Med. 374 (25) (2016) 2453-2464.
[43] O. Miotto, et al., Genetic architecture of artemisinin-resistant Plasmodium falciparum, Nat. Genet. 47 (3) (2015) 226-234.

[44] M. Imwong, et al., The spread of artemisinin-resistant Plasmodium falciparum in the greater Mekong subregion: a molecular epidemiology observational study, Lancet Infect. Dis. 17 (5) (2017) 491-497.

[45] F. Huang, et al., A single mutation in K13 predominates in southern China and is associated with delayed clearance of Plasmodium falciparum following artemisinin treatment, J. Infect. Dis. 212 (10) (2015) 1629-1635.

[46] E. Talundzic, et al., Selection and spread of artemisinin-resistant alleles in Thailand prior to the global artemisinin resistance containment campaign, PLoS Pathog. 11 (4) (2015) e1004789.

[47] M. Imwong, et al., An outbreak of artemisinin resistant falciparum malaria in eastern Thailand, Sci. Rep. 5 (2015) 17412.

[48] K. Thriemer, et al., Delayed parasite clearance after treatment with dihydroartemisinin-piperaquine in Plasmodium falciparum malaria patients in Central Vietnam, Antimicrob. Agents Chemother. 58 (12) (2014) 7049-7055. 